\title{
Efficient adaptive phase field simulation of dendritic growth in a forced flow at low supercooling
}

\author{
C.W. Lan*, C.M. Hsu, C.C. Liu \\ Department of Chemical Engineering, National Taiwan University, Taipei 10617, Taiwan, ROC \\ Received 28 November 2001; accepted 18 March 2002 \\ Communicated by D.T.J. Hurle
}

\begin{abstract}
Efficient phase field simulation using an adaptive finite volume method is presented for two-dimensional dendritic growth in a forced flow. The extremely large domain (over $2^{18}$ in the ratio of cell size), while keeping very small cell size at the interface, makes the simulation at low supercooling possible. With a strong forced flow, the upstream tip speed reaches a steady state quickly even at low supercooling. The upstream arm develops side branches easily, but remains similar after rescaling, i.e., self-affine, as the diffusive one at steady state. (C) 2002 Elsevier Science B.V. All rights reserved.
\end{abstract}

PACS: $68.70 .+\mathrm{w} ; 81.30 . \mathrm{Fb}$

Keywords: A1. Computer simulation; A1. Dendrites; A1. Morphological instability; A1. Fluid flows; A1. Heat transfer; A1. Phase field model

\section{Introduction}

The development of microstructures is important in solidification processing. Extensive experimental observations of dendritic growth have been performed using transparent organic materials under normal or reduced gravity [1-3]. The effect of forced convection has also been investigated [4]. When the supercooling is low, the growth can be characterized by the steady dendrite tip speed, radius, and shape. This thus makes the comparison with theory possible. The solvability theory

\footnotetext{
*Corresponding author. Fax: + 886-2-2363-3917.

E-mail address: cwlan@ccms.ntu.edu.tw (C.W. Lan).
}

[5] provides a background for understanding of the dendrite evolution and its pattern selection. However, the details of the structure development require a global model and this renders the numerical solution of a complete time-dependent Stefan problem. Solving the problem using front tracking is extremely challenging due to the large deformation of the dendrite shape.

With the progress of phase field simulation [6-8], the simulation of dendritic growth has been greatly simplified. The phase filed introduces a continuous phase field variable $\phi(\boldsymbol{r})$ to describe the interface through a rapid transition of the function. Nevertheless, the phase field simulation is not exactly the same as the front tracking unless the 
sharp interface limit, i.e., the interface thickness being much smaller than the capillary one, can be approached. Karma and Rappel [6] provided simple criteria of choosing parameters that the sharp interface limit can be released to the order of the capillary length. This makes the phase field simulation much more solvable by using a simple structured mesh. Unfortunately, due to the use of the structured mesh, the computation effort, being about $L_{\mathrm{D}}^{3}$, increases dramatically as the domain size $\left(L_{\mathrm{D}}\right)$ increases, because the mesh size for the interface needs to be small enough for the sharp interface. For a large supercooling $\Delta$ (e.g., $\Delta=-0.55)$, the dendrite tip speed reaches a steady state quickly before the far thermal field becomes affected. As a result, the computational domain can be quite small. Good agreement with the prediction of the solvability theory has been obtained easily [6]. However, the reliable experimental observations were performed at low supercoolings [3]. At a low supercooling (e.g., $\Delta=-0.1)$, the dendrite tip growth rate is small (small growth Peclet number) and it takes a long time to reach a steady state. To avoid the far field being affected, for the comparison with the solvability theory, the domain size for simulation needs to be increased dramatically. Provatas et al. [7] provided a heuristic criterion for the domain size required (about $5 \sim 10 D / V_{\mathrm{t}}$ ) for a realistic simulation and the time $\left(9 D / V_{\mathrm{t}}^{2}\right)$ for reaching a steady state, where $D$ is the dimensionless thermal diffusivity and $V_{\mathrm{t}}$ the dimensionless tip speed. They proposed an efficient adaptive finite element method for simulation and the domain area was up to $102,400 \times 51,200$, where the ratio of the largest to the smallest cell size was $2^{17}$. More importantly, their computing cost scaled with the domain area $\left(L_{\mathrm{D}}^{2}\right)$. Full three-dimensional (3D) simulation of dendritic growth has also been proposed recently [8]. However, it was performed for diffusive growth only.

On earth, convection cannot be avoided and the buoyancy convection can significantly affect the dendritic growth. In materials processing, to enhance composition uniformity, a forced flow is usually added purposely. Bouissou and Pelce [9] have extended the solvability theory, based on a parabolic dendrite, to predict quantitatively the velocity selection in a forced uniform flow; their result is referred as the Oseen-Ivantsov solution. Scaling laws characterizing the tip operating state have also been proposed. Unfortunately, the existing experiments [4,10-11] produced different results from their theory; discrepancy existed even at the tip speed. Therefore, similar to the works for diffusive growth, the phase field simulation of dendritic growth in a forced flow is highly desired to examine the theory, before it is used for the comparison with experiments. Adaptive mesh was considered by Tonhardt and Amberg [12]. However, they considered a shear flow passing a seed on a wall and thus the comparison with the theory was not possible. Recently, Tong et al. [13] have made this attempt to simulate the velocity and tip shape selection under a forced convection, but at a high supercooling $(\Delta=-0.55)$, using structure meshes. The maximum domain area used was only about $1024 \times 2048$. If the dendrite tip radius is obtained from a parabolic function fitting to the overall dendrite head, their calculations agree well with the Oseen-Ivantsov solution. The selection parameter was found to be independent of the flow rate. However, due to the use of small domains, their calculations cannot be extended to low supercoolings. Indeed, as they mentioned, the simulation at low supercoolings, higher flow velocities, and in $3 \mathrm{D}$ remain a great challenge for the future.

In this report, we present an efficient phase field simulation based on an adaptive finite volume method. Since an extremely large domain $(204,800 \times 102,400)$ and cell-size ratio (up to $2^{18}$ ) are used and the computational cost also scales linearly with the domain area, we are able to simulate the dendritic growth at low supercoolings in a forced flow, even at high flow velocities. This opens a new window for realistic simulation of dendritic growth and makes the comparison with the theory possible. Our domain size is greater than that reported by Provatas et al. [7] and the velocities larger than that used by Tong et al. [13], and this is not an upper limit. All the calculations are performed efficiently in a personal computer (Pentium-III/800 with 512M SRAM) and the simulation approach can be extended to 3D easily. 


\section{Phase field simulation}

For comparison purposes, the dendritic growth is simulated using a phase field model employed in [7]. The temperature is rescaled to $\theta=C_{\mathrm{p}}$ $\left(T-T_{\mathrm{m}}\right) / \Delta H$, where $C_{\mathrm{p}}$ is the specific heat of solid, $T_{\mathrm{m}}$ the melting point, and $\Delta H$ the heat of fusion. The phase field variable $\phi$ is set to be 1 in solid, -1 in melt, and 0 at the interface. The time $t$ is rescaled by $\tau_{0}$, which characterizes atomic movement, the length is rescaled by $w_{0}$, which characterizes the interface thickness, and the velocity is rescaled by $\alpha / w_{0}$, where $\alpha$ is the dimensional thermal diffusivity. Then, the equations for temperature and phase field variable are as follows:

$$
\begin{gathered}
\frac{\partial \theta}{\partial t}+D \boldsymbol{\nabla} \boldsymbol{v} \theta=D \boldsymbol{\nabla}^{2} \theta+\frac{1 \partial \phi}{2 \partial t}, \\
w^{2} \frac{\partial \phi}{\partial t}=\boldsymbol{\nabla}\left(w^{2} \boldsymbol{\nabla} \phi\right)+\left[\phi-\lambda \theta\left(1-\phi^{2}\right)\right]\left(1-\phi^{2}\right) \\
-\frac{\partial}{\partial x}\left(w \frac{\partial w}{\partial \beta} \frac{\partial \phi}{\partial y}\right)+\frac{\partial}{\partial y}\left(w \frac{\partial w}{\partial \beta} \frac{\partial \phi}{\partial x}\right),
\end{gathered}
$$

where $D$ is the dimensionless thermal diffusivity and $\boldsymbol{v}$ the velocity; $w=1+\varepsilon \cos 4 \beta$ and $\varepsilon$ is a parameter for the interface energy anisotropy; $\beta=$ $\tan ^{-1}[(\partial \phi / \partial y) /(\partial \phi / \partial x)]$. The calculation of the velocity requires the treatment of two-phase flow at the interface, and the model proposed by Beckermann et al. [14] is also adopted here. The conservation equations for mass and momentum are as follows, respectively,

$\boldsymbol{\nabla} \boldsymbol{v}=0$,

$\frac{1}{D} \frac{\partial v}{\partial t}+\boldsymbol{\nabla}(\boldsymbol{v} \boldsymbol{v})=\operatorname{Pr} \boldsymbol{\nabla}^{2} \boldsymbol{v}-\boldsymbol{\nabla} P+\boldsymbol{F}$,

where $\operatorname{Pr}=v / \alpha$ is the Prandtl number and $\boldsymbol{F}$ accounts the dissipative force between two phases; $\operatorname{Pr}=23.1$ is used, which is about the value for succinonitrile $(\mathrm{SCN})$. The interactive force $\boldsymbol{F}=$ $-0.5 C \operatorname{Pr}\left[(\phi+1) / w_{0}\right]^{2} \boldsymbol{v}$, where $C$ is an empirical constant. Beckermann et al. [14] used the analytical result of the Poiseville flow between two plates to fit the model and got $C=2.757$. To solve these equations, the SIMPLE scheme and momentum interpolation are further used for pressure/ velocity coupling on the collocated cells. Extensive benchmark comparison of the numerical imple- mentation for the fluid flow calculation can be found elsewhere [15]. The Gauss-Siedel method is used for inner iterations of all variables. The simulation starts at a small seed in a large domain, which corresponds to $2 \mathrm{D}$ uniform lattices having $2^{18} \times 2^{17}$ cells. A sample mesh during growth is shown in Fig. 1, where we have enlarged the interface region into three different scales. As shown, mesh refinement is performed by subdividing uniformly each parent cell into four kid cells. As such, detailed substructures can also be described nicely if the mesh level is large enough; in Fig. 1, 18 levels are used. Two criteria are used for the refinement. One is based on the phase field variable and one for the temperature (e.g., no less then 9 levels of cells for $0.1 \Delta$ ); the later is used only when the secondary flow is important. In addition, the level difference for adjacent cells is also restricted to one, and this improves significantly the accuracy of the method. By mapping the grid into a quad-tree data structure, the dynamic pointer functions in FORTRAN90 can be fully

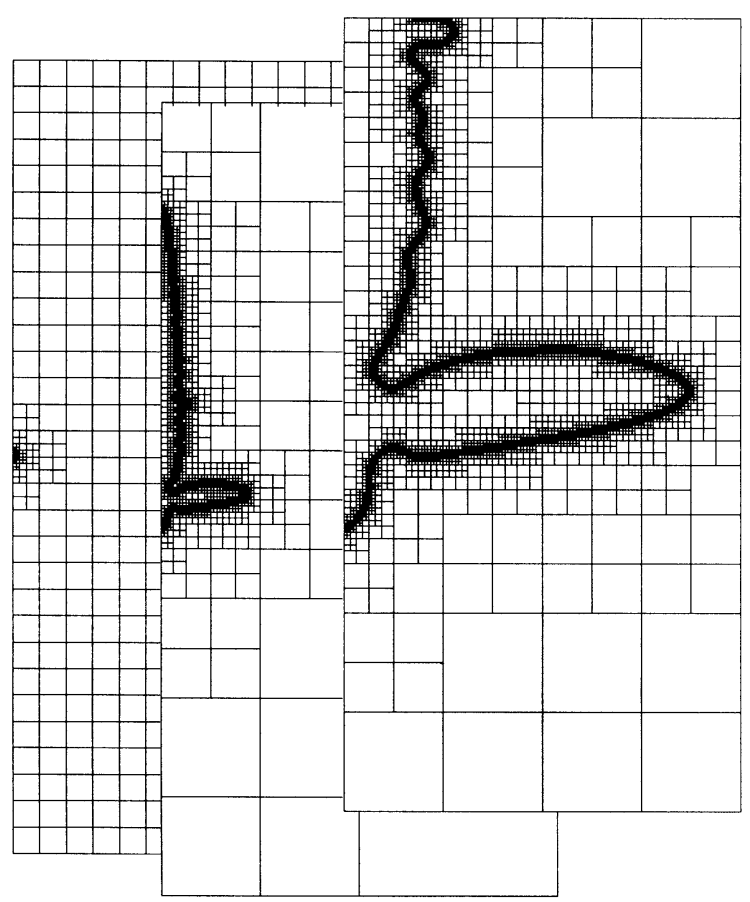

Fig. 1. Adaptive mesh for dendritic growth in a forced flow; local magnifications of the mesh are also shown. 
adopted and this makes the programming straightforward. The implicit Euler scheme is used for time integration and this allows larger integration timesteps to be used. Nevertheless, the limitation for the timesteps is that the interface advancement needs to be inside the refined zone. Otherwise, numerical instability can cause problems. In the present simulation, the CPU time for a growth scales about $L_{\mathrm{D}}^{2}$. In other words, the computational cost is about linearly proportional to the domain area. This is comparable to the performance of the scheme proposed by Provatas et al. [7].

\section{Results and discussion}

For the cases without convection, i.e., the diffusive growth, we have obtained good agreement with the prediction by the solvability theory for various supercoolings. Some calculated results and comparison with previous works are summarized in Table 1 . In Table $1, d_{0}$ is the capillary constant and its value is about $2 \times 10^{-7} \mathrm{~cm}$ for $\mathrm{SCN}$, while the $w_{0}$ is the reference length. Since the minimum cell size is smaller than the reference length, say $0.1 w_{0}$, and the length scale for the whole domain is more than $10^{5}$, it is indeed required an extremely large ratio of the biggest and the smallest cell sizes for a realistic simulation if one would like to consider both the global heat flow and the local branching behavior. As shown in Table 1, for high supercoolings, excellent agreement with previous results is obtained. However, a small discrepancy is found for $\Delta=$ -0.25 from that predicted by Provatas et al. [7]. In our calculations, the calculated tip speed is only slightly less than the theoretical value, while the value calculated by Provatas et al. is slightly higher. It is believed that the value being slightly smaller is due to the growth of side arms. With the existence of side arms, the surrounded thermal fields are warmer than that without side arms. As a result, the slightly slower tip speed is expected; the solvability theory is developed for a single dendrite. For $\Delta=-0.1$, the steady state is still not reached in $10^{5} \tau_{0}$. The time evolution of the tip speed for diffusive growth is shown by the dashed line in Fig. 2; we have purposely used the $\log -\log$ scale to show the asymptotic decreasing rate. The slow decay rate of tip speed $\left(V_{\mathrm{t}} \sim t^{-0.22}\right)$ is similar to that calculated by Provatas et al. $\left(V_{\mathrm{t}} \sim t^{-0.27}\right)$ [7]. Many different domain sizes for calculations are also examined. In general, if the far field is not

Table 1

Summarized calculated results and comparison with previous predictions

\begin{tabular}{|c|c|c|c|c|c|c|c|}
\hline \multirow[t]{2}{*}{$\Delta$} & \multirow[t]{2}{*}{$U\left(w_{0} / \tau_{0}\right)$} & \multirow[t]{2}{*}{$d_{0} / w_{0}$} & \multirow[t]{2}{*}{$L_{\mathrm{D}}$} & \multicolumn{2}{|c|}{ Tip speed $V_{t}\left(\alpha / d_{0}\right)$} & \multicolumn{2}{|c|}{ Self-affine parameters } \\
\hline & & & & This study & Previous study $[6-7,9]$ & $\beta$ & $\eta$ \\
\hline-0.55 & 0 & 0.139 & 128 & 0.0171 & 0.017 & 1.1951 & 0.4805 \\
\hline-0.3 & 0 & 0.055 & 1000 & 0.000664 & 0.00064 & 0.9976 & 0.5146 \\
\hline-0.25 & 0 & 0.043 & 6400 & $0.000265^{*}$ & 0.00027 & $0.9131^{\mathrm{a}}$ & $0.5018^{\mathrm{a}}$ \\
\hline-0.1 & 0 & 0.01846 & 102,400 & $7.1 \times 10^{-6^{* * *}}$ & $7.2 \times 10^{-6}$ & $0.7871^{\mathrm{b}}$ & $0.4239^{\mathrm{b}}$ \\
\hline-0.55 & 1.0 & 0.139 & 512 & 0.0240 & $0.0244[13]$ & & \\
\hline-0.25 & 2.0 & 0.043 & 12,800 & $1.08 \times 10^{-3}$ & $1.1 \times 10^{-3 \mathrm{c}}[9]$ & 1.0693 & 0.5065 \\
\hline \multirow[t]{4}{*}{-0.1} & 10.0 & 0.01846 & 204,800 & $1.92 \times 10^{-4}$ & $1.97 \times 10^{-4 \overline{\mathrm{d}}}[9]$ & 1.058 & 0.4452 \\
\hline & 10.0 & 0.01846 & 6400 & $1.89 \times 10^{-4}$ & & & \\
\hline & 10.0 & 0.01846 & 3200 & $2.05 \times 10^{-4}$ & & & \\
\hline & 10.0 & 0.01846 & 1600 & $2.30 \times 10^{-4}$ & & & \\
\hline
\end{tabular}

${ }^{*} t=6000 \tau_{0} ;{ }^{* *} t=110000 \tau_{0}$.

${ }^{\mathrm{a}} \beta=0.97$ and $\eta=0.55$ in Ref. [17].

${ }^{\mathrm{b}} \beta=0.73$ and $\eta=0.43$ in Ref. [17].

${ }^{\mathrm{c}} R=4.33$.

${ }^{\mathrm{d}} R=4.65$ (the radius curvatures are obtained from the parabolic fitting of the dendritic head; $\frac{1}{3}$ of the length). 


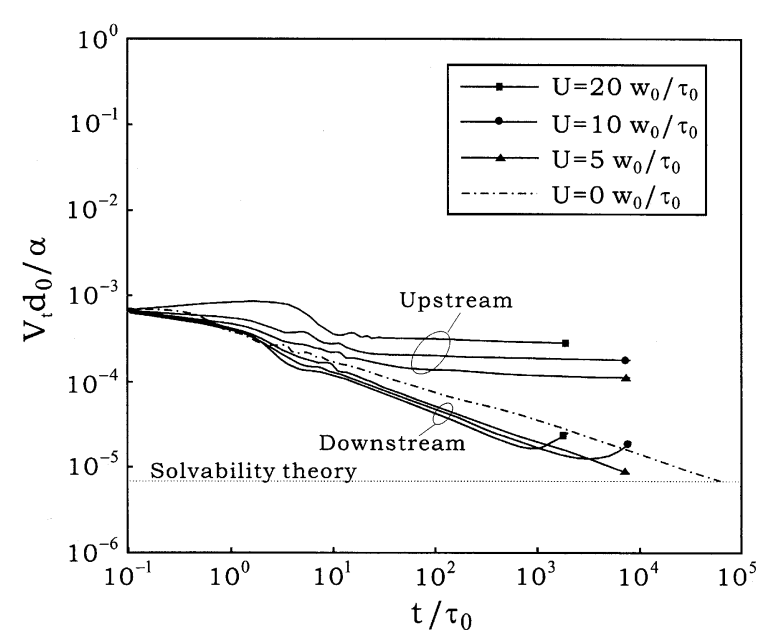

Fig. 2. Time evolution of tip speeds (upstream and downstream) at different flow rates; $\Delta=-0.1$.

affected, the domain size will not affect the tip speed. However, for low supercoolings, such as $\Delta=-0.1$ and -0.25 , it requires much longer time for a steady state and thus an extremely large domain to avoid the far field from being affected. For $\Delta=-0.1$, due to the extremely long integration time (larger than $10^{5} \tau_{0}$ ), the domain area at least being $102,400 \times 102,400$ (quarter of domain) is required.

From the simulation of diffusive growth, it seems to be quite discouraging for a realistic simulation at low supercoolings. In addition to the extremely large domain, the time for reaching a steady state is extremely long. Interestingly, once the flow is imposed, it is found that the tip speed is increased and the steady-state tip speed at upstream can be reached much more quickly. Fig. 2 also shows the tip speeds at upstream and downstream, respectively, for three external flow speeds ( $U=5,10$, and $\left.20 w_{0} / \tau_{0}\right)$. As shown, the time for reaching a steady state for the upstream tip is significantly reduced and it decreases with the increasing flow rate; the time for reaching to a steady state is only about $10^{2 \sim 3} \tau_{0}$. If we use $D / V_{\mathrm{t}}^{2}$ for estimation, the result here is reasonable because the upstream tip speed is increased about one to two orders in magnitude. For $\Delta=-0.25$, the time for reaching a steady state at the same flow rate is about $10^{3} \tau_{0}$, while the diffusive growth takes more than $10^{4} \tau_{0}$ to reach a steady state. Nevertheless, the tip speeds of the other side arms are still changing with time, especially at the downstream. Some thermal (left) and flow (right) fields and dendrite shapes (left) are shown in Fig. 3 for three different times at $U=10 w_{0} / \tau_{0}$; the dendrite shapes at three different times $\left(t=4000,6000\right.$ and $\left.8000 \tau_{0}\right)$ are plotted to be about the same size for comparison. As shown, the thermal boundary layer in front of the upstream tip is much thinner. As a result, the growth rate is the highest there. More interestingly, due to the flow, as shown, the effect of side arm is suppressed; the isotherms are pushed toward the side arm. Therefore, the upstream tip seems to grow independently like a single dendrite. The downstream arm shows some interesting behavior. At the beginning, the downstream tip grows slowly, and its asymptotic decreasing rate is about the same as the diffusive one (also see Fig. 2). However, when the side arm is grown large enough, at high convection velocity, there is a boundary layer separation near its side tip and a wake (back flow) is induced. As a result, the tip speed of the down stream arm is increased due to the back flow; the isotherms behind the dendrite are pulled toward the dendrite, as shown in Fig. 3. With a higher flow rate, the wake occurs earlier and downstream speed also bounds back earlier. Furthermore, with a strong forced flow, since the time for the upstream tip to reach a steady state is significantly shorter, the domain for calculation can be reduced dramatically as well. In fact, in addition to the estimated size by $5 \sim 10 D / V_{\mathrm{t}}$ for a diffusive growth, one has to take the flow boundary layer into account as well. Therefore, $10 \mathrm{v} / V_{\mathrm{t}}$ can be further adopted as the second criterion for estimating the domain size. In the present calculation, we have found that the reduction of domain more than 10 times to $(6400 \times 3200)$ gives about the same result, as shown in Table $1 ; L_{\mathrm{D}}$ is the domain size. This indeed encourages the feasibility of extension of present calculation to 3D because the domain and time can be reduced significantly.

We further compare our upstream tip speeds with the Oseen-Ivantsov solution [9], and as 

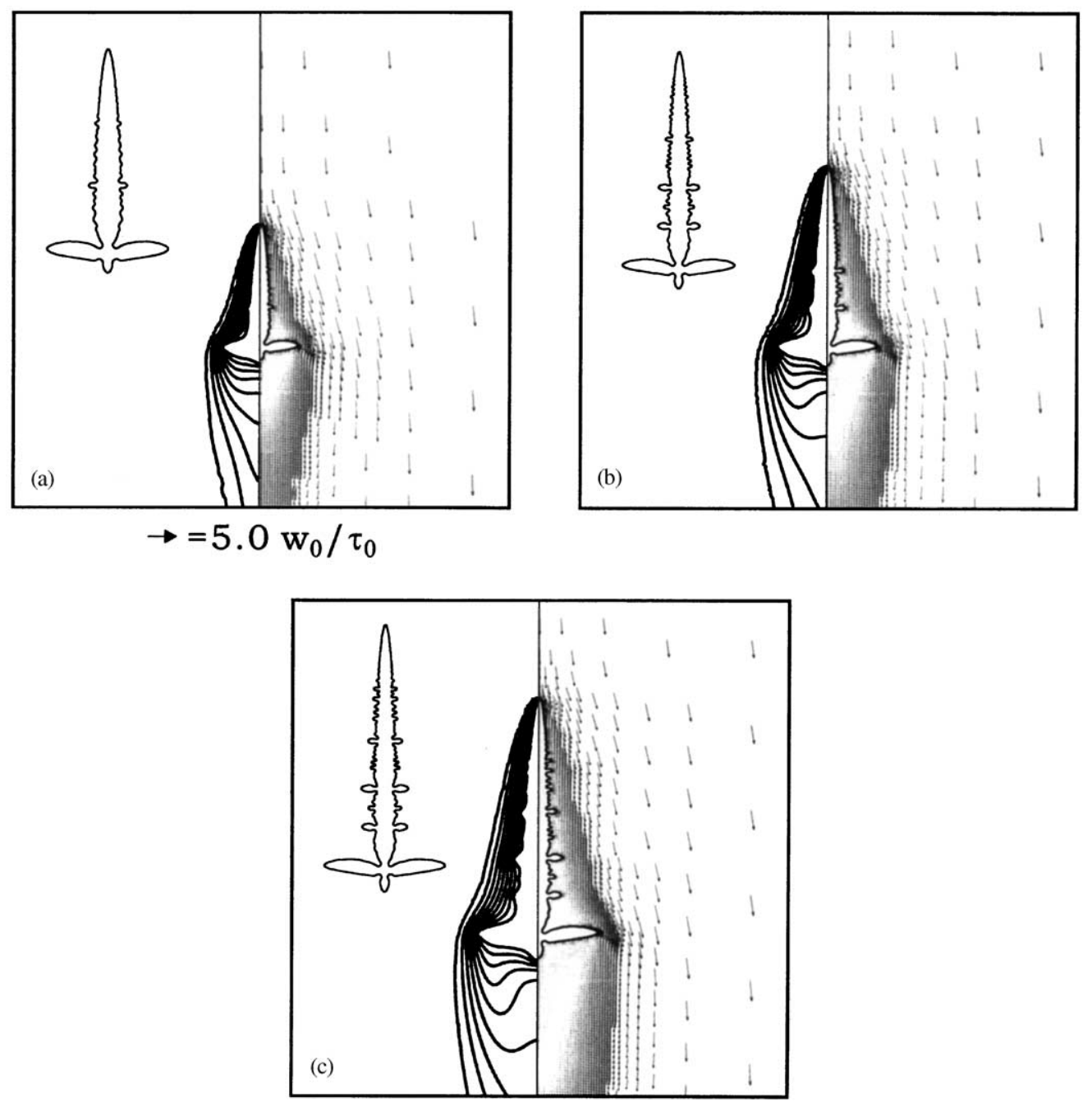

Fig. 3. Isotherms (left), flow patterns (right), and whole dendrite shapes at different times: (a) $t=4000 \tau_{0} ;$ (b) $6000 \tau_{0}$; (c) $8000 \tau_{0}$, $\Delta=-0.1$ and $U=10 w_{0} / \tau_{0}$.

shown in Table 1 for $\Delta=-0.25$ and -0.1 , the agreement is reasonably good if the tip radius is obtained from a parabolic fitting of the dendrite head (about $\frac{1}{3}$ of the arm length). Further comparison is also performed for $\Delta=-0.55$ with that by Tong et al. [13]. As shown in Table 1, good agreement is obtained as well. Because the OseenIvantsov solution neglected the interfacial tension, the local tip speed, which has been modified by the interfacial energy, cannot be used for comparison. The local tip radius decreases with the increasing external flow rate; the local tip is regarded as the top $5 \%$ of the dendrite head.

In addition, as shown in Fig. 3 for $U=10 w_{0} / \tau_{0}$, many side branches have been induced at the upstream arm, while the other arms remain smooth. This is believed to be the cause of numerical noises [7], which are amplified by the flow. For the same mesh, we have not yet found any side branches for diffusive growth. Clearly, increasing the flow rate induces more side branches. The use of finer mesh may delay slightly 


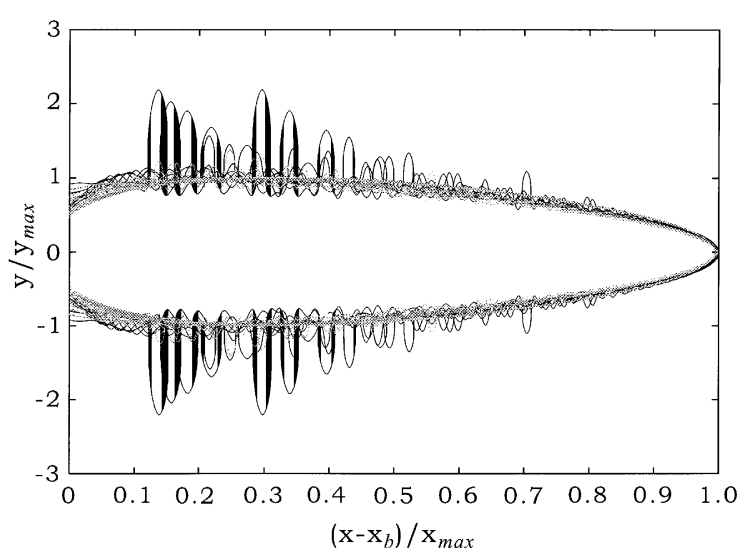

Fig. 4. Scaled dendrite profiles (solid lines) for $\Delta=-0.1$ at nine different times after the crossover; the gray lines are the other results (both diffusive and convective growth) having the scaling parameters shown in Table 1.

the onset of the side branches. Similar side branching due to the flow was also observed in previous experiments [16] and simulation [12].

More importantly, beside the side branching, the overall dendrite shape remains to be selfaffine. If we take the shapes at different times ranging from 2000 to $8000 \tau_{0}$, and rescale $x$ by $\left(x-x_{\mathrm{b}}\right) / x_{\max }$ and $y$ by $y / y_{\max }$, as shown in Fig. 4 (solid lines), the overall dendrite shapes are about the same; $x_{\mathrm{b}}$ is the base position, $x_{\max }$ the maximum arm length, and $y_{\max }$ the dendrite width. If $x_{\max }$ is proportional to $t^{\beta}$ and $y_{\max }$ to $t^{\eta}$, then the best fitting gets $\beta \sim 1$ and $\eta \sim 0.5$, as shown in Table 1 . This scaling result can be easily understood. Since both the velocity $V_{\mathrm{t}} \sim \mathrm{d} x / \mathrm{d} t \sim \beta t^{\beta-1}$ and the radius of curvature $R \sim\left(\mathrm{d}^{2} x / \mathrm{d} y^{2}\right)^{-1} \sim t^{2 \eta-\beta}$ are constant at steady state, we shall get $\beta=1$ and $\eta=0.5$. More importantly, the shape seems to be universal and is affected very little by the side branches. Even for diffusive growth, if the steady state can be reached, we obtain similar results, as shown in Table 1. More interestingly, the exponents of $\beta=1$ and $\eta=0.5$ indicate that the shape remains to be parabolic, even though the local tip is not. Because the tip head remains to be parabolic, the results thus agree reasonably well with the Oseen-Ivantsov solution. Furthermore, if the results of all the steady-state cases for both diffusive and convective growth are put together, the self-affine shape is again universal and affected very little by the supercooling and convection; besides that for $\Delta=-0.1$ and $U=10 w_{0} / \tau_{0}$, other shapes are represented by gray lines. For the case of no flow for $\Delta=-0.1$, at the same period time (but without a steady state), as shown in Table 1, the exponents are about 0.79 for $\beta$ and 0.42 for $\eta$. These values are in good agreement with those obtained by Dantzig et al. [17] $(\beta=0.73$ and $\eta=0.43$ ). Although the calculations here are only 2D, the calculation approach can be extended to 3D easily. Due to the much shorter time for reaching a steady-state tip speed at the upstream, the domain for calculation can be significantly reduced. As a result, the steady upstream tip speed of 3D dendritic growth at low supercoolings is much more computable.

\section{Conclusion}

We have presented an efficient adaptive phase simulation for dendritic growth in a uniform forced flow at low supercooling. Extensive comparison with previous studies is also performed, and good agreement is found. Due to the faster growth speed and the suppression of the influence from the side arms, the upstream dendrite tip tends to grow independently and reaches a steady growth quickly. This, for the first time, allows the comparison with the theory possible for low supercooling. If the tip radius is obtained from the global dendrite tip, the calculated upstream tip speed agrees well with the Oseen-Ivantsov solution. Interestingly, the flow around the upstream tip easily induces side branching, while other branches remain smooth. This is also consistent with experimental observation. More importantly, regardless the side branches, the rescaled dendrite shape is hardly affected by the flow and the supercooling. In other words, the self-affine dendrite shape remains to be universal at the steady state.

\section{Acknowledgements}

C.W.L would like to thank Prof. R.F. Sekerka for introducing this topic. A careful review of the 
manuscript and comments by Prof. G. Amberg are highly appreciated. This research is sponsored by the National Science Council of the Republic of China.

\section{References}

[1] S.C. Huang, M.E. Glicksman, Acta Metall. 29 (1981) 701.

[2] M.E. Glicksman, Mater. Sci. Eng. 65 (1984) 45.

[3] M.E. Glicksman, Microgravity News, NASA 4 (1997) 4.

[4] Y.W. Lee, R. Ananth, W.N. Gill, J. Crystal Growth 132 (1993) 226.

[5] J.S. Langer, in: J. Souleties, J. Vannenimus, R. Stora (Eds.), Chance and Matter, Lectures in the Theory of Pattern Formation, Les Houches Session XL VI, NorthHolland, Amsterdam, 1987. p. 629.
[6] A. Karma, W.J. Rappel, Phys. Rev. E 53 (1996) R3017; A. Karma, W.J. Rappel, Phys. Rev. E 57 (1998) 4323.

[7] N. Provatas, N. Goldenfield, J. Dantzig, Phys. Rev. Lett. 80 (1998) 3308.

[8] A. Karma, W.J. Rappel, Phys. Rev. Lett. 77 (1996) 4050.

[9] Ph. Bouissou, P. Pelce, Phys. Rev. A 40 (1989) 6673.

[10] S. Dash, W.N. Gill, Int. J. Heat Mass Transfer 27 (1984) 1345.

[11] R. Ananth, W.N. Gill, J. Crystal Growth 91 (1988) 587.

[12] R. Tonhardt, G. Amberg, J. Crystal Growth 213 (2000) 161.

[13] X. Tong, C. Beckermann, A. Karma, Phys. Rev. E 61 (2000) 49.

[14] C. Beckermann, H.-J. Diepers, I. Steinbach, A. Karma, X. Tong, J. Comp. Phys. 154 (1999) 468.

[15] C.W. Lan, C.C. Liu, C.M. Hsu, J. Comp. Phys., in press.

[16] N. Noel, H. Jamgotchian, B. Billia, J. Crystal Growth 181 (1997) 117.

[17] N. Provatas, N. Goldendeld, J. Dantzig, Phys. Rev. Lett. 83 (22) (1999) 4496. 